\title{
X-Ray Diffraction from Periodically Patterned GaAs Nanorods Grown onto GaAs[111]B
}

\author{
ANTON DAVYDOK, ANDREAS BIERMANNS, ULLRICH PIETSCH, \\ JÖRG GRENZER, HENDRIK PAETZELT, and VOLKER GOTTSCHALCH
}

\begin{abstract}
We present a high-resolution X-ray diffraction pattern of periodic GaAs nanorod (NR) ensembles and individual GaAs NRs grown catalyst-free throughout a prepatterned amorphous $\mathrm{SiN}_{x}$ mask onto GaAs[111]B surfaces. The experiments were performed at a home laboratory using synchrotron radiation in combination with a micron-sized beam prepared by compound refractive lenses. The structural properties were probed by measuring RSMs $\left(q_{x}, q_{z}\right)$ in the vicinity of GaAs(111) and (222) reflections. Besides the GaAs substrate peak, we found a second peak referring to NRs with lattice mismatch of 0.23 pct with respect to the substrate, probably caused by structural defects. The lateral periodicity of NRs was probed by $q_{x}$ scans, and the NR height obtained from the width of the diffraction curve along $q_{z}$. Grazing-incidence in-plane diffraction revealed the appearance of small crystallites of cubic $\gamma-\mathrm{Si}_{3} \mathrm{~N}_{4}$ caused by recrystallization of $\mathrm{SiN}_{x}$ during NR growth. Whereas measurements at the home diffractometer provided average structure parameters, the micron-sized X-ray beam experiment was used to probe the parameters at individual NRs.
\end{abstract}

DOI: $10.1007 / \mathrm{s} 11661-009-9868-3$

(C) The Minerals, Metals \& Materials Society and ASM International 2009

\section{INTRODUCTION}

SEMICONDUCTOR nanorods (NRs) grown by vapor-liquid-solid (VLS) epitaxy are of particular interest for the creation of materials with new electronic and optical properties ${ }^{[1]}$ Exploiting quantum effects, one is able to tune the emission wavelength and to induce onedimensional (1-D) electronic transport. In the VLS mode, NRs are grown onto [111] planes of a zinc-blend or diamond-type semiconductors by solution from a molten eutectic alloy formed by a metallic seed, with the diameter and position of grown NRs crucially depending on the statistical process of the metallic droplet formation. The spatial position and the diameter of the molten seeds (typically, $\mathrm{Au}$ ) determine the position and size of the grown NRs onto the substrate. Due to the statistical character of both quantities, the NRs are nonuniform and rather randomly located at the substrate.

One route to prepare NRs of uniform diameter and defined inter-rod spacing is the use of prepatterned

ANTON DAVYDOK and ANDREAS BIERMANNS, Postdoctoral Students, and ULLRICH PIETSCH, Professor and Solid State Group Leader, are with Solid State Physics, Siegen University, 57068 Siegen, Germany. JÖRG GRENZER, Scientist, is with FZ-Dresden Rossendorf, 013414 Dresden, Germany. HENDRIK PAETZELT, Postdoctoral Student, is with the Leibniz Institute for Surface Modification, Ion Beam Technology Department, 04318 Leipzig, Germany. VOLKER GOTTSCHALCH, Scientist, is with Solid State Chemistry, University of Leipzig, 04103 Leipzig, Germany.

This article is based on a presentation given in the symposium entitled "Neutron and X-Ray Studies for Probing Materials Behavior," which occurred during the TMS Spring Meeting in New Orleans, LA, March 9-13, 2008, under the auspices of the National Science Foundation, TMS, the TMS Structural Materials Division, and the TMS Advanced Characterization, Testing, and Simulation Committee. Article published online June 16, 2009 substrates. ${ }^{[2]}$ This can be performed via selective areametal organic vapor phase epitaxy (SA-MOVPE), where the NRs are grown from small circular openings defined by electron-beam lithography and wet chemical etching of a thin $\mathrm{SiN}_{x}$ layer. ${ }^{[3]}$ Using opening diameters in the range of a few hundred nanometers and inter-rod distances in the range of several microns, uniformly sized NRs were grown. ${ }^{[4]}$

One key problem for the understanding of NR growth in such arrays is the mutual interaction of growing NRs with the $\mathrm{SiN}_{x}$ mask, which can result in alloy formation or recrystallization of the initially amorphous $\mathrm{SiN}_{x}$.

Structure characterization of individual NRs is typically performed by high-resolution transmission electron microscopy at single NRs. ${ }^{[5]}$ In addition, X-ray diffraction has been used to obtain structural information from a statistical ensemble of nonuniform NRs. ${ }^{[6,7]}$ Nowadays, the achievements in X-ray optics to define an intense coherent X-ray beam with submicron diameter allows for three-dimensional (3-D) characterization of individual nano-objects using coherent diffraction imaging (CDI) ${ }^{[7-9]}$ However, strain analysis by CDI of individual nano-object is still a challenging task. ${ }^{[10]}$

The aim of this work was to measure the average and individual properties of GaAs NRs grown in periodic arrays by catalyst-free SA-MOVPE throughout a prepatterned $\mathrm{SiN}_{x}$ mask onto GaAs[111]B surfaces.

The samples were characterized using coplanar highresolution X-ray diffraction in a home laboratory and by coplanar X-ray diffraction at the European Synchrotron Radiation Facility (ESRF) using the microfocus setup at the ID1 beamline. The latter method can provide spatially resolved information about the shape of individual NRs within the NR pattern. 


\section{SAMPLE PREPARATION}

The structures have been grown onto [111]B oriented GaAs substrate covered by a 15 -nm-thick amorphous $\mathrm{SiN}_{x}$ layer. The silicon nitride has been deposited by plasma-enhanced chemical vapor deposition at $300{ }^{\circ} \mathrm{C}$. Within an area of $250 \times 250 \mu \mathrm{m}^{2}$, the $\mathrm{SiN}_{x}$ layer was partially removed by electron beam lithography, defining an ordered array of circular openings with diameters of $600 \mathrm{~nm}$ in an electron-sensitive resist followed by wet chemical etching using $\mathrm{NH}_{4} \mathrm{~F}: \mathrm{HF}: \mathrm{H}_{2} \mathrm{O}$ solution. The selective-area growth was carried out using low-pressure (50 mbar) MOVPE in an AIXTRON AIX200 reactor (Aachen, Germany). The total flow into the reactor amounted to $7 \mathrm{slm}$. Thrimethylgallium $(\mathrm{TMGa}=3.75 \mathrm{~mL})$ and $\operatorname{arsine}\left(\mathrm{AsH}_{3}=50 \mathrm{~mL}\right)$ were used as group-III and group-V materials. The growth temperature was set to $750{ }^{\circ} \mathrm{C}$, providing equally hexagonally shaped NRs with uniform spacing. ${ }^{[4]}$

Two samples have been investigated with different inter-rod spacing, $D$ : sample 1 with $D=1.25 \mu \mathrm{m}$ and sample 2 with $D=3 \mu \mathrm{m}$. Prior to X-ray measurements, the NR arrays were inspected by scanning electron microscopy (SEM). Figure 1 presents SEM pictures of both samples, verifying the uniformity of hexagonally shaped NRs. Sample 1 is a hexagonal array of NRs with diameters of about $700 \mathrm{~nm}$ and heights of $500 \mathrm{~nm}$ (Figure 1(a)); sample 2 is a square-shaped array with NR diameters of about $600 \mathrm{~nm}$ and heights of $260 \mathrm{~nm}$ (Figure 1(b)). At few positions NRs are missing due to incomplete openings in the mask.

\section{X-RAY CHARACTERIZATION OF THE ENTIRE NR ARRAY}

Prior to the synchrotron experiment, all samples were characterized using a high-resolution diffractometer GE HR-XRD 3003 at Siegen University using a coplanar Bragg reflection and $\mathrm{Cu} K_{\alpha 1}$ radiation. Reciprocal space maps (RSMs) have been recorded close to the GaAs(111) Bragg reflection by subsequent $\omega+\Delta \omega-2 \Theta$ scans for different offset angles $\Delta \omega$. After recording, the angular values were recalculated into energy-independent coordinates of reciprocal space using the relations
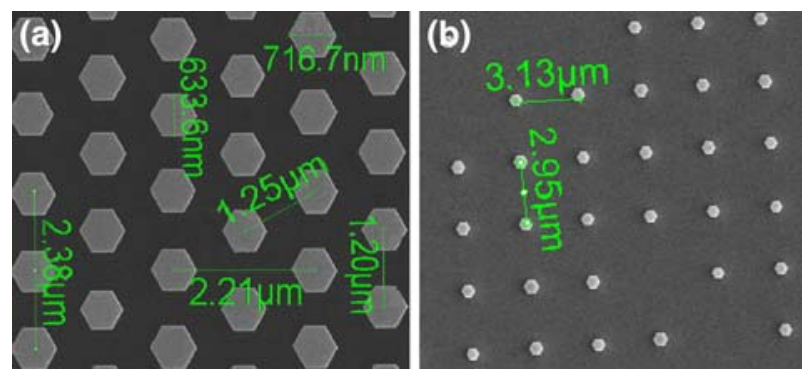

Fig. 1-SEM picture of $\mathrm{GaAs}[111]+15 \mathrm{~nm} \mathrm{SiN}_{x}+\mathrm{GaAs}$ NR sample grown on GaAs[111]B substrate: (a) $D=1.2 \mu \mathrm{m}$ and $(b)$ $D=3 \mu \mathrm{m}$.

$$
\begin{aligned}
& q_{z}=2 \pi / \lambda(\sin \omega+\sin (2 \theta-\omega)) \\
& q_{x}=2 \pi / \lambda(\cos \omega-\cos (2 \theta-\omega))
\end{aligned}
$$

where $\lambda$ is the wavelength, and $\omega$ and $(2 \theta-\omega)$ are the angles of incident and reflected beams with respect to the diffracting lattice plane parallel to the surface.

Grazing-incidence diffraction measurements were taken along the in-plane coordinate

$$
q_{\|}=2 \pi / \lambda\left(\sin \theta_{i}+\sin \theta_{f}\right)
$$

where $\theta_{i}$ and $\theta_{f}$ are the angles of incident and reflected beams with respect to the lattice planes perpendicular to the surface.

Figure 2(a) shows the RSM of sample 1. Two distinct maxima are visible along $q_{z}$. The peak at $q_{z}=19.255 \mathrm{~nm}^{-1}$ (peak 1) corresponds to GaAs(111). Apart from the substrate peak (1), a second peak (2) is visible displaced by $\Delta q_{z}=0.045 \mathrm{~nm}^{-1}$, indicating tensile expansion of 0.23 pct. Both peaks are accompanied by sets of satellite peaks equally spaced by $\Delta q_{x}=0.0025 \mathrm{~nm}^{-1}$, measuring an inter-rod distance of $D=2 \pi / \Delta q_{x}=2.5 \mu \mathrm{m}$. Due to the arbitrary azimuthal alignment of the sample at the goniometer, this length measures the inter-rod distance between second neighbored NRs, as seen in Figure 1(a). The same sample was measured again after removal of the $\operatorname{SiN}_{x}$ layer (as subsequently discussed). The respective RSM (Figure 2(b)) shows the two peaks along $q_{z}$, but only peak 2 is accompanied by satellite reflections. Here, the azimuthal alignment differs from that of the first experiment. By chance, the distance $\Delta q_{x}=0.0053 \mathrm{~nm}^{-1}$ measures the next nearest neighbor distance $D=$ $1.18 \mu \mathrm{m}$. Whereas the full-width at half-maximum (FWHM) $\Delta q_{z}$ of the substrate reflection (peak 1) along the vertical direction is small and reflects the instrumental resolution of the experiment, the FWHM $\Delta q_{z}$ of peak 2 provides a height $2 \pi / \Delta q_{z}$ of about $420 \mathrm{~nm}$, which
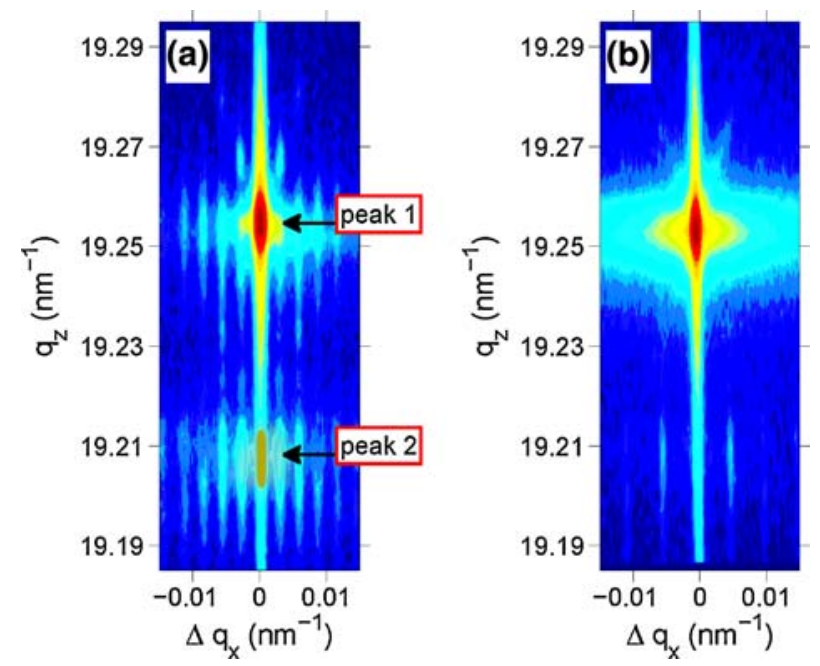

Fig. 2-RSM of GaAs NRs taken at the GaAs(111) reflection in the high-resolution diffractometer GE HR-XRD $3003(a)$ before and (b) after removal of $\operatorname{SiN}_{x}$. 
agrees fairly well with the average height of NRs. Therefore, peak 2 can be associated with scattering from NRs. Unfortunately, no satellite peaks could be resolved on maps from sample 2 with $D=3 \mu \mathrm{m}$ caused by the fact that the coherence length of the laboratory diffractometer, roughly estimated to be $1.5 \mu \mathrm{m}$, is much smaller than the inter-rod distance. That fact has motivated measurements with synchrotron radiation.

\section{CHARACTERIZATION OF THE WHOLE NR ARRAY USING SYNCHROTRON RADIATION}

The experiments have been performed at beamlines ID1 and ID10b of ESRF. The high intensity and the small divergence of the incidence beam motivated measurements of the whole NR array using the method of X-ray grazing incidence diffraction (GID). Here, the incident beam strikes the samples under a shallow angle $\alpha_{i}$ with respect to the sample surface, and the diffracted beam is measured in a direction perpendicular to the surface normal of the sample. Subsequently, GID measures lattice planes perpendicular to the NR main axis. One of these lattice planes is (4-2-2) using zincblend notation. Exploiting the effect of total external reflection of the X-ray beam at the air-sample interface, the penetration depth can be extremely reduced to a few nanometers in the case where $\alpha_{i}$ is smaller then the critical angle $\alpha_{c}$ of total external reflection. ${ }^{[11]}$ For the wavelength $\lambda=0.12399 \mathrm{~nm}, \alpha_{c}$ is $0.3 \mathrm{deg}$. For the purpose of detection, we used a $1-\mathrm{D}$ position sensitive detector (PSD) aligned parallel to the surface normal.

The bottom curve in Figure 3 shows a wide in-plane $q_{\|}$scan through sample 1 using an incidence angle $\alpha_{i}=0.2$ deg. Beginning from (4-2-2), several other Bragg reflections are visible, which can be identified by GaAs with either cubic or wurzite structure.

These reflections appear because of randomly oriented crystallites grown onto the amorphous $\mathrm{SiN}_{x}$ layer in between the perfectly aligned NRs. In addition, the

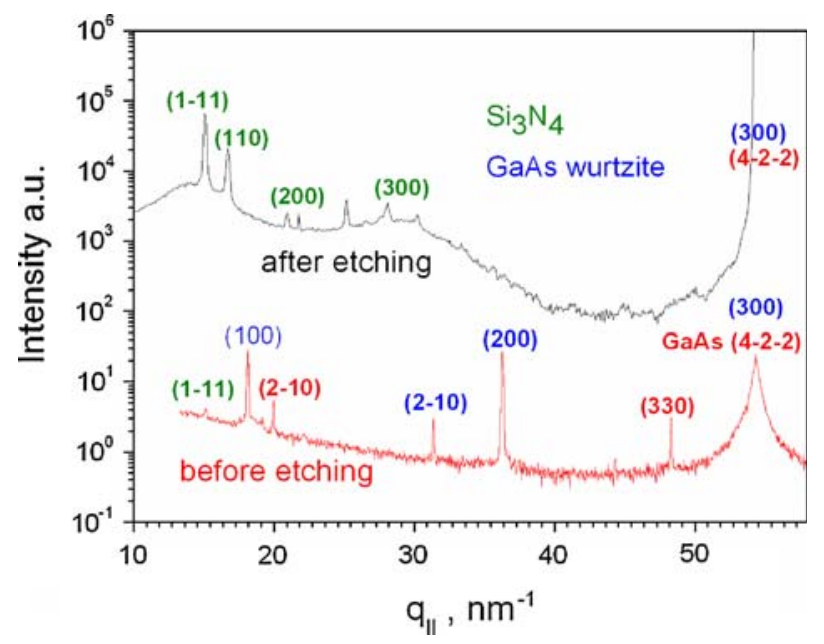

Fig. 3- q\|-line scans measured from samples before and after removing $\mathrm{SiN}_{x}$. Blue indices correspond to wurzite structure GaAs, and green indices are cubic $\gamma-\mathrm{Si}_{3} \mathrm{~N}_{4}$. peak at $q_{\|}=15.13 \mathrm{~nm}^{-1}$ can be indexed by the crystalline $\gamma-\mathrm{Si}_{3} \mathrm{~N}_{4}$ (1-11) reflection. Unfortunately, no further peaks are visible because of the high instrumental background.

In order to clarify the question of whether peak 2 in Figure 2 could be originated from $\gamma-\mathrm{Si}_{3} \mathrm{~N}_{4}$ as well, we recorded the same in-plane line scan after chemical removal of amorphous $\mathrm{SiN}_{x}$ using a solution of $\mathrm{NH}_{4} \mathrm{~F}$ (top curve in Figure 3). Again, one can see the (4-2-2) reflection, but no further GaAs peaks. These peaks have been removed by the etching process. However, this scan has been performed using a different experimental setup, providing much higher intensity and lower noise compared to the first experiment. Because of this, the structure of the background can be resolved, providing several peaks that can be identified with crystalline $\gamma-\mathrm{Si}_{3} \mathrm{~N}_{4}$, in addition to the (1-11). They reveal a lattice parameter, which is slightly compressed by about 3 pct compared to the literature value. ${ }^{[12]}$

Finally, we measured the average shape function of the GaAs NRs using the GID setup. This was done by recording an in-plane RSM with coordinates $q_{x}$ and $q_{y}$. Due to the GID scheme, the X-ray beam probes the whole NR pattern and, subsequently, the RSM represents the average shape of illuminated NRs. As seen in Figure 4, six different crystal truncation rods can be identified referring to the hexagonal symmetry of the NRs.

\section{X-RAY DIFFRACTION FROM INDIVIDUAL NRs}

Nowadays, the ID1 beamline at ESRF provides the possibility of using a microfocus setup for diffraction measurements defined by a set of compound refractive lenses, focusing the incident beam to a spot size ${ }^{[16]}$ of about $500 \times 1500 \mathrm{~nm}^{2}$ in the present case. Using coplanar-diffraction geometry, the beam spot onto the sample equals roughly the inter-rod spacing of sample 1, but it allows for single NR measurement in the case of sample 2. Using this setup, we recorded RSMs of individual NRs close to (111) and (222) Bragg reflection

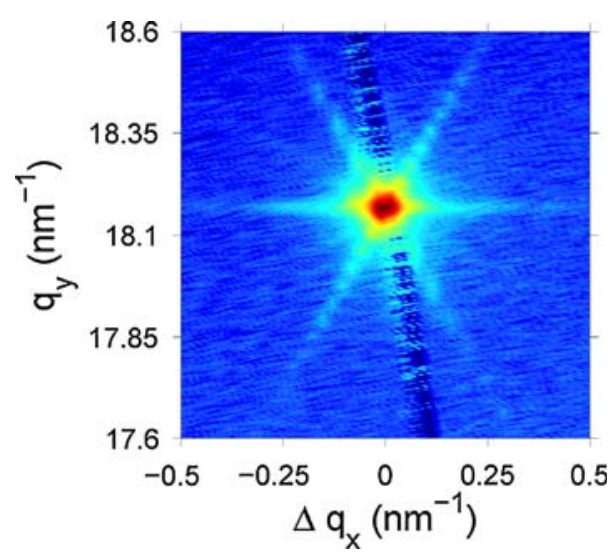

Fig. 4-RSM of GaAs NRs taken at (4-2-2) reflection in GID geometry. 
(a)
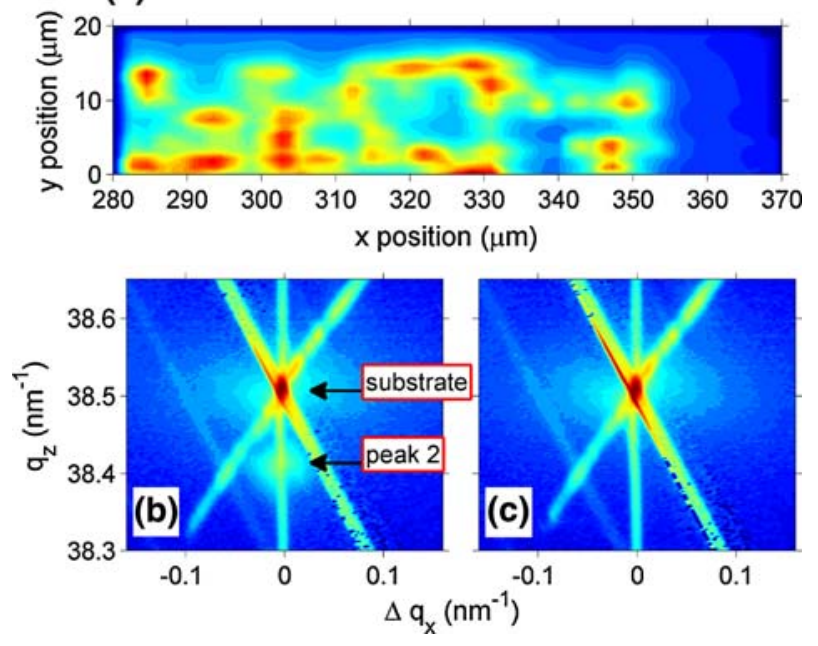

Fig. 5-Intensity pattern of diffuse scattering recorded inside the NR pattern close to the (222) Bragg peak. (a) RSMs taken (b) inside and (c) outside the patterned area.

of GaAs using a beam energy of $10 \mathrm{keV}$ and a 1-D position sensitive detector.

The patterned area was selected by screening the diffuse scattering in the vicinity of the GaAs Bragg peak. ${ }^{[13,14]}$ Using an angular position fixed at about 1 deg off with respect to the exact Bragg peak position, the intensity of diffuse scattering varied by a factor of more than 5 inside and outside the patterned area. In addition, the diffuse scattering changed by a factor of 2 at particular NR positions compared to a position in between two NRs. This fingerprint allowed us to scan a certain area of the patterned sample and to identify individual NRs. The mapping of diffuse scattering inside such an area is shown in Figure 5(a). It is evident that the maxima of diffuse scattering are distributed similar to the NR pattern shown in Figure 1(b) (distances between peaks on the map are in the range from 2.76 to $3.12 \mu \mathrm{m})$. Subsequently, we have associated the scattering maxima with certain NR positions. Due to the screening scheme used, it is very likely that the detected intensity maxima correspond to the edges or corners of individual NRs where the diffuse scattering is strong. This may explain why certain NR positions are missing in the map. Furthermore, due to the angle-dependent size of the footprint, we cannot exclude that two NRs are excited simultaneously. However, RSM maps recorded inside and outside the NR array are clearly different. Two examples of RSMs in the vicinity of the GaAs(222) reflection are shown in Figures 5(b) and (c). The RSM taken inside the NR array (b) shows again the peak associated to the NRs, as already measured for the whole NR ensemble (Figure 2). This peak is not present outside the array (Figure 5(c)). At the same time, RSMs taken nominally between individual NRs show lower intensity of peak 2. By taking $q_{z}$ scans from individual RSMs, one can estimate the heights of respective individual NRs from the FWHM of peak 2. They are revealed to be nonuniform and our measurements provide a variation of heights ranging from 310 to $480 \mathrm{~nm}$.
One has to note that the crossed lines and the perpendicular line in Figures 5(b) and (c) are artifacts of the experiment (monochromator and PSD streaks) For further explanation, see. ${ }^{[15]}$ Also, the additional two oblique lines on the left are artifacts of the experimental setup.

\section{DISCUSSION}

We analyzed GaAs NRs grown on GaAs[111]B by high-resolution X-ray diffraction. Using coplanar diffraction in a home laboratory, we measured the average structure parameters of the whole NR array. The microfocus setup at ESRF was used to probe the structure parameters of individual NRs.

In both experiments, we have seen a second Bragg peak close to the GaAs one. Using microfocus, we could verify that this peak appears only at positions of GaAs NRs. Therefore, peak 2 was associated with scattering from NRs. The measured lattice expansion with respect to the substrate could be explained by inclusions of atoms with larger covalent radius compared to $\mathrm{Ga}$ and As. However, after SEM and EDX inspection, we can exclude any contamination of another element with concentration larger then 0.5 pct. Thus, the measured lattice mismatch must originate from the influence of structural defects such as decorated stacking faults, for example. Indeed, $q_{z}$ scans taken from the RSM of individual NRs revealed different lattice mismatches, which may refer to different numbers of such defects. Unfortunately, for this study, we were not able to probe individual NRs by use of asymmetric diffractions, which are necessary for detailed phase analysis. ${ }^{[16]}$

One should note that the appearance of the lattice mismatch was very advantageous for our experiment, because low intense structural features could be identified outside the high-intense substrate diffraction.

Grazing-incidence diffraction at the whole NR ensemble revealed peaks of crystalline $\gamma-\mathrm{Si}_{3} \mathrm{~N}_{4}$, which might be grown also during the growth process. Obviously, they cannot be removed by the etching process, which is sensitive to amorphous $\mathrm{SiN}_{x}$. These $\mathrm{Si}_{3} \mathrm{~N}_{4}$ crystals are grown with random orientation with respect to the sample surface. Using this suggestion, peak 2 in Figures 2 and 5 could be indexed by the (200) Bragg reflection of $\gamma-\mathrm{Si}_{3} \mathrm{~N}_{4}$ as well, but has to show nearly constant intensity and changing $q_{x}$. Because the peak is sharp, this suggestion can be ruled out.

The crystallization of $\mathrm{Si}_{3} \mathrm{~N}_{4}$ crystallites probably takes place at the growth temperature of NRs or at the recooling process after the growth. From the FWHM of peaks related to $\mathrm{Si}_{3} \mathrm{~N}_{4}$ shown in Figure 4, the crystallite size can be estimated to be $32 \mathrm{~nm}$, which is much smaller than the NR diameter. One may suggest that these crystallites grow preferentially at the interface between the growing GaAs NR and the amorphous $\mathrm{SiN}_{x}$ layer to release the interface strain. The appearance of strain is visible by satellite peaks close to the substrate, which is originated by scattering from drilled holes within the $\mathrm{SiN}_{x}$ mask, as shown in Figure 2(a). This explains why these satellites disappear after etching the $\mathrm{SiN}_{x}$ layer (Figure 2(b)). 
Finally, one has to note that our nanofocus experiment was not ideal because we could not measure an entire NR. This is caused by the limited resolution, which did not fit the size of the object, but also by the fact that we did not tune the beam spot to the center of an individual NR. Using a nanofocus diameter similar to the site of nano-objects, one can characterize the whole 3-D shape of individual NRs by CDI. ${ }^{[16,17]}$ Our sample system promises revelation of the details of the different shapes and defect structures of heteroepitaxial NRs.

\section{ACKNOWLEDGMENT}

The authors thank Ana Diaz and T.H. Metzger, ID1 beamline at ESRF, for support.

\section{REFERENCES}

1. C. Thelander, P. Agarwal, S. Brongersma, J. Eymery, L.F. Feiner, A. Forchel, M. Scheffler, W. Riess, B.J. Ohlsson, U. Gösele, and L. Samuelson: Mater. Today, 2006, vol. 9, pp. 28-35.

2. J. Fan, P. Werner, and M. Zacharias: Small, 2006, vol. 2, pp. 70017

3. K. Ikejiri, J. Noborisaka, S. Hara, J. Motohisa, and T. Fukui: J. Cryst. Growth, 2007, vol. 298, pp. 616-19.
4. H. Paetzelt, V. Gottschalch, J. Bauer, G. Benndorf, and G. Wagner: J. Cryst. Growth, 2008, vol. 310, pp. 5093-97.

5. M.W. Larson, J.B. Wagner, M. Wallin, P. Hakansson, L.E. Fröberg, L. Samuelson, and L.R. Wallenberg: Nanotechnology, 2007, vol. 18, pp. 015504-12.

6. B. Mandl, J. Stangl, T. Martensson, A. Mikkelson, J. Eriksson, L. Karlsson, G. Bauer, L. Samuelson, and W. Seifert: Nano Lett., 2006, vol. 6, pp. 1817-21.

7. J. Eymery, F. Rieutord, V. Favre-Nicolin, O. Robach, Y.-M. Niquet, L. Fröberg, T. Mårtensson, and L. Samuelson: Nano Lett., 2007, vol. 7, pp. 2596-2601.

8. V. Chamard, J. Stangl, S. Labat, B. Mandl, R.T. Leichner, and T.H. Metzger: J. Appl. Cryst., 2008, vol. 41, pp. 272-80.

9. M. Hanke, M. Dubslaff, and M. Schmidbauer: Appl. Phys. Lett., 2008, vol. 92, pp. 193109-1-193109-3.

10. R. Harder, M.A. Pfeifer, G.J. Williams, I.A. Vartanyants, and I.K. Robinson: Phys. Rev. B, 2007, vol. 76, pp. 115425-1-115425-4

11. H. Dosch: Critical Phenomena at Surfaces and Interfaces, vol. 149, Springer Tracts in Modern Physics, Springer Verlag, Berlin, 1992.

12. S.J. Patil, K.C. Mohite, A.B. Mandale, M.G. Takwale, and S.A. Gangal: Surf. Coat. Technol., 2005, vol. 200 (7), pp. 2058-64.

13. A. Davydok, A. Biermanns, U. Pietsch, J. Grenzer, H. Paetzelt, V. Gottschalch, and J. Bauer: Phys. Status Solidi, in press.

14. C. Mocuta, J. Stangl, K. Mundboth, T.H. Metzger, G. Bauer, I.A. Vartanyants, M. Schmidbauer, and T. Boeck: Phys. Rev. B, 2008, vol. 77, pp. 245425-1-245425-6.

15. U. Pietsch, V. Holy, and T. Baumbach: Advanced Physics, Springer, New York, NY, 2005.

16. A. Biermanns, A. Davydok, U. Pietsch, H. Paetzelt, V. Gottschalch, and T.H. Metzger: unpublished research.

17. J. Miao, P. Charalambous, J. Kirz, and D. Sayre: Nature, 1999, vol. 400 , pp. $342-44$. 